\title{
Afzelin attenuates asthma phenotypes by downregulation of GATA3 in a murine model of asthma
}

\author{
WENBO ZHOU and XIUHONG NIE \\ Department of Respiratory Diseases, Xuanwu Hospital, Capital Medical University, Beijing 100053, P.R. China
}

Received April 4, 2014; Accepted December 19, 2014

DOI: $10.3892 / \mathrm{mmr} .2015 .3391$

\begin{abstract}
Asthma is a serious health problem causing significant mortality and morbidity globally. Persistent airway inflammation, airway hyperresponsiveness, increased immunoglobulin E (IgE) levels and mucus hypersecretion are key characteristics of the condition. Asthma is mediated via a dominant T-helper 2 (Th2) immune response, causing enhanced expression of Th2 cytokines. These cytokines are responsible for the various pathological changes associated with allergic asthma. To investigate the anti-asthmatic potential of afzelin, as well as the underlying mechanisms involved, its anti-asthmatic potential were investigated in a murine model of asthma. In the present study, BALB/c mice were systemically sensitized using ovalbumin (OVA) followed by aerosol allergen challenges. The effect of afzelin on airway hyperresponsiveness, eosinophilic infiltration, Th2 cytokine and OVA-specific IgE production in a mouse model of asthma were investigated. It was found that afzelin-treated groups suppressed eosinophil infiltration, allergic airway inflammation, airway hyperresponsiveness, OVA-specific IgE and Th2 cytokine secretion. The results of the present study suggested that the therapeutic mechanism by which afzelin effectively treats asthma is based on reduction of Th2 cytokine via inhibition of GATA-binding protein 3 transcription factor, which is the master regulator of Th2 cytokine differentiation and production.
\end{abstract}

\section{Introduction}

Asthma is a chronic inflammatory airway disease. It affects over 300 million individuals worldwide with an expected increase of 100 million by $2025(1,2)$. The pathophysiological characteristics of allergic asthma, including chronic pulmonary eosinophilia, airway hyperresponsiveness (AHR) to a variety of nonspecific spasmogenic stimuli, excessive airway mucus

Correspondence to: Dr Xiuhong Nie, Department of Respiratory Diseases, Xuanwu Hospital, Capital Medical University, 45 Changchun Street, Beijing 100053, P.R. China

E-mail: niexiuhong@gmail.com

Key words: asthma, immunoglobulin E, airway hyperresponsiveness, T-helper 2, eosinophil, GATA-binding protein 3 production and elevated serum immunoglobulin E (IgE) levels are all associated with aberrant T-helper 2 (Th2) cell responses. Th2 cells are known to secrete interleukin (IL)-4, -5, -9 and -13 . These cytokines, particularly IL $-4,-5$ and -13 , have been documented to have a relatively important role in asthma progression. Th2 cell differentiation is driven by the transcription factor GATA-binding protein 3 (GATA-3), a member of the GATA family of zinc finger proteins (3). This transcription factor is known as the master regulator of Th2-cell differentiation. GATA-3 is suppressed by T-bet expressed in T cells, a Th1-specific transcription factor, which is hypothesized to induce interferon (IFN) $-\gamma$ production while inhibiting IL-4 production (4).

Afzelin (Fig. 1) is a flavonol glycoside found in Ficus palmata and Nymphaea odorata. Previously, it has been found to inhibit lipid peroxidation and cyclooxygenase (COX)-1 and COX-2 in vivo. It is the rhamnoside of kaempferol, which has been documented to suppress inflammatory-cell infiltration in a mouse model of asthma (5). A previous study indicated that afzelin inhibits the growth of breast cancer cells through stimulating apoptosis, while being relatively non-toxic to normal cells (6). However, the effects of afzelin on asthma phenotypes have remained to be elucidated. The present study was performed to investigate the anti-asthmatic effect of afzelin and its mechanism of action in a mouse model of asthma.

\section{Materials and methods}

Experimental animals. A total of 30 female BALB/c mice (five weeks old, 25-30 g) were attained from the animal house of the Capital Medical University (Beijing, China), and maintained under controlled conditions, temperature $\left(24 \pm 2^{\circ} \mathrm{C}\right)$, relative humidity $(60 \pm 10 \%)$ and photoperiod (12-h light/dark cycle). The room was well ventilated ( $>10$ air changes $/ \mathrm{h}$ ) with fresh air, as per the Committee for the Purpose of Control and Supervision on Experiments on Animals guidelines. Animals were fed on a standard pellet diet and sterilized water was provided ad libitum. Animals acclimated for seven days were used for the pre-clinical studies. Approval of the animal experimental protocols was obtained from the ethics committee of the Capital Medical University (Beijing, China).

Reagents. Chicken egg albumin (OVA, grade V), aluminium hydroxide gel (alum) and dexamethsone (Dexa), acetyl- $\beta$-methylcholine chloride (methacholine) and protease 
inhibitor cocktail were purchased from Sigma-Aldrich (St. Louis, MO, USA). Antibodies used for western blotting were purchased from Cell Signaling Technology (Beverly, MA, USA). Afzelin (purity, 99\%) was acquired from Chirochem (Daejeon, Korea). All other chemicals and reagents were commercially obtained from Sigma-Aldrich and were of the highest quality.

Segregation of animals and dosing schedule. Mice were segregated into six groups (six mice in each group) following acclimation; each group was termed according to sensitization/challenge/treatment: Group 1, SHAM/phosphate-buffered saline (PBS)/Vehicle (Veh; normal controls); group 2, OVA/OVA/Veh (OVA controls, OVA-sensitized and OVA-challenged); group 3, OVA/OVA/Dexa [OVA-sensitized, OVA-challenged and Dexa-treated $(0.75 \mathrm{mg} / \mathrm{kg})]$; and groups 4-6,OVA/OVA/afzelin [OVA-sensitized, OVA-challenged and afzelin-treated (0.1, 1 and $10 \mathrm{mg} / \mathrm{kg}$ )]. The test compounds and the Dexa were administered orally, once daily from day 19 to day 23 (Fig. 2) (7). PBS was used as a vehicle.

Sensitization, airway OVA challenging and treatment. The animals were sensitized intraperitoneally with $40 \mu \mathrm{g}$ OVA plus $2.6 \mathrm{mg}$ aluminum hydroxide in $200 \mu \mathrm{l}$ PBS on days 0 and 7. Mice were then challenged from days 19 to 23 (5 min per day) with 5\% OVA in PBS (OVA groups) or PBS (Sham/PBS/Veh) as described previously with certain modifications (8). Mice were administered the test drug and Dexa once a day from days 19 to 23 . Mice were sacrificed on day 24 by heart puncture under ether anesthesia (Sigma-Aldrich), and bronchoalveolar lavage was performed to evaluate lung eosinophilia.

Evaluation of AHR. AHR, in the form of airway resistance was estimated in anesthetized mice using the FlexiVent system (Synol High-Tech, Beijing, China), which uses a computer-controlled mouse ventilator and integrates with respiratory mechanics, as described previously (9). Final results were expressed as airway resistance with increasing concentrations of methacholine (Mch; 0, 2, 4, 8, 12 and $16 \mathrm{mg} / \mathrm{ml})$.

Bronchoalveolar lavage fluid (BALF) collection. After mice were bled and sacrificed following anesthesia with ether, BALF was collected for differential cell counting and measurement of cytokines. This was performed by cannulating the upper part of the trachea and lavaging three times with $0.5 \mathrm{ml}$ PBS containing $0.05 \mathrm{mM}$ EDTA (7). The BALF was centrifuged at $4,000 \times \mathrm{g}$ at $4^{\circ} \mathrm{C}$ for $3 \mathrm{~min}$ and the cells were separated from the fluid. The supernatant was stored at $-70^{\circ} \mathrm{C}$ until use. The cells were re-suspended in PBS containing $0.05 \mathrm{mM}$ EDTA and the total cell number was determined by using a hemocytometer. The differential BAL cells were counted using microscopy (MCL-3000; MCALON, Beijing, China) following cytospin preparations and Giemsa staining (Giemsa stain modified, Sigma-Aldrich).

Cytokine measurement. Cytokine measurement was performed from serum samples of animals. Levels of

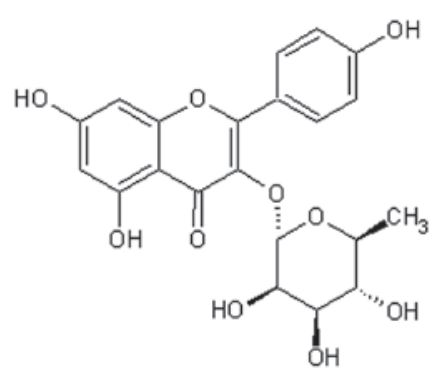

Figure 1. Structure of afzelin; 5,7-dihydroxy-2-(4-hydroxyphenyl)-3-[(2S $3 R, 4 R, 5 R, 6 S)-3,4,5$-trihydroxy-6-methyloxan-2-yl] oxychromen-4-one); molecular mass, $432.38 \mathrm{~g} / \mathrm{mol}$.

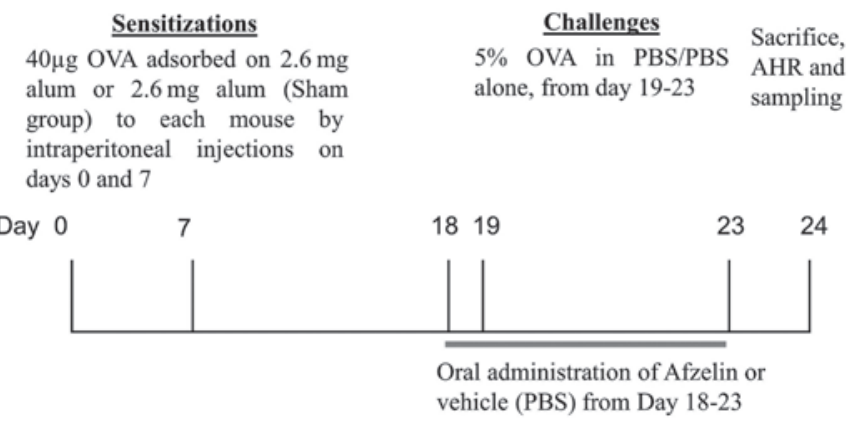

Figure 2. Experimental protocol for the induction of allergic asthma. Female BALB/c mice (5 weeks old) were grouped, sensitized and challenged. OVA, chicken egg albumin; PBS, phosphate-buffered saline.

cytokines IL-5, -13 and -4 as well as IFN- $\gamma$ were determined using ELISA (R\&D Systems, Minneapolis, MN, USA). The ELISAs were performed as per the manufacturer's instructions.

Measurement of OVA-specific IgE. Each well of a microtiter plate (Abcore, Ramona, CA, USA) was coated with $5 \mu \mathrm{g}$ OVA in $100 \mu \mathrm{l}$ PBS overnight at $4^{\circ} \mathrm{C}$. Following three washes, nonspecific sites were blocked with $0.5 \%$ Tween 20 (Abcore) in PBS. Mouse sera in duplicate were added to the Ag-coated wells, the plates were incubated and bound $\operatorname{IgE}$ was detected with biotinylated anti-mouse IgE (BD Pharmingen, San Diego, CA, USA). Streptavidin-peroxidase conjugates (Takara Biotechnology Co., Ltd., Dalian, China) were added and the bound enzymes were detected with the addition of a tetramethylbenzidine substrate system (BD Pharmingen) and absorbance was read at $450 \mathrm{~nm}$ using an ultraviolet spectrophotometer (UV-3600; Shimadzu Corporation, Kyoto, Japan). Absorbance was converted to arbitrary units.

Western blot analysis. The lungs were homogenized in a homogenizing buffer [1\% NP- $40,150 \mathrm{mM} \mathrm{NaCl}$, $50 \mathrm{mM}$ 4-(2-hydroxyethyl)-1-piperazineethanesulfonic acid (Sigma-Aldrich), phenylmethylsulfonyl fluoride and complete protease inhibitor cocktail (Bio-Rad Laboratories, Inc., Hercules, CA, USA)]. Protein estimation of the samples was performed according to the Bradford method (10). For western blotting, $30 \mu \mathrm{g}$ protein was denatured at $100^{\circ} \mathrm{C}$ for $5 \mathrm{~min}$ in Tris-glycine SDS (Abcore) sample loading buffer. Protein samples were loaded onto $10 \%$ SDS gels and resolved at $70 \mathrm{~V}(300 \mathrm{~mA})$ for $3 \mathrm{~h}$ and then electro-transferred onto a 
Table I. Effect of afzelin on total cell count and differential cell count.

\begin{tabular}{|c|c|c|c|c|c|}
\hline \multirow[b]{2}{*}{ Treatment } & \multirow[b]{2}{*}{ Total count $\left(\times 10^{4} / \mathrm{ml}\right)$} & \multicolumn{4}{|c|}{ Differential count $(\%)$} \\
\hline & & Macro & Mono & Eosino & Neutro \\
\hline SHAM & $3.1 \pm 1.1$ & $51.3 \pm 5.5$ & $49.6 \pm 4.5$ & - & - \\
\hline OVA/OVA/Veh & $49.2 \pm 10.0$ & $7.4 \pm 1.1$ & $13.6 \pm 4.0$ & $61.7 \pm 4.9$ & $12.9 \pm 3.3$ \\
\hline Dexa $(0.7$ mg/kg) & $23.5 \pm 8.3$ & $26.8 \pm 7.6$ & $31.9 \pm 13.0$ & $22.3 \pm 11.0$ & $8.0 \pm 3.8$ \\
\hline Afzelin $(0.1 \mathrm{mg} / \mathrm{kg})$ & $35.6 \pm 11.2$ & $17.9 \pm 6.9$ & $38.2 \pm 2.3$ & $52.2 \pm 4.4$ & $9.4 \pm 2.3$ \\
\hline Afzelin $(1 \mathrm{mg} / \mathrm{kg})$ & $17.7 \pm 6.3$ & $38.9 \pm 3.3$ & $32.2 \pm 2.7$ & $15.9 \pm 1.5$ & $5.3 \pm 1.2$ \\
\hline Afzelin $(10 \mathrm{mg} / \mathrm{kg})$ & $15.5 \pm 10.2$ & $47.0 \pm 13.0$ & $42.5 \pm 9.3$ & $11.5 \pm 3.2$ & $3.3+1.1$ \\
\hline
\end{tabular}

Values are expressed as the mean \pm standard error of the mean. Dexa, dexamethasone; OVA, chicken egg albumin; Veh, vehicle.

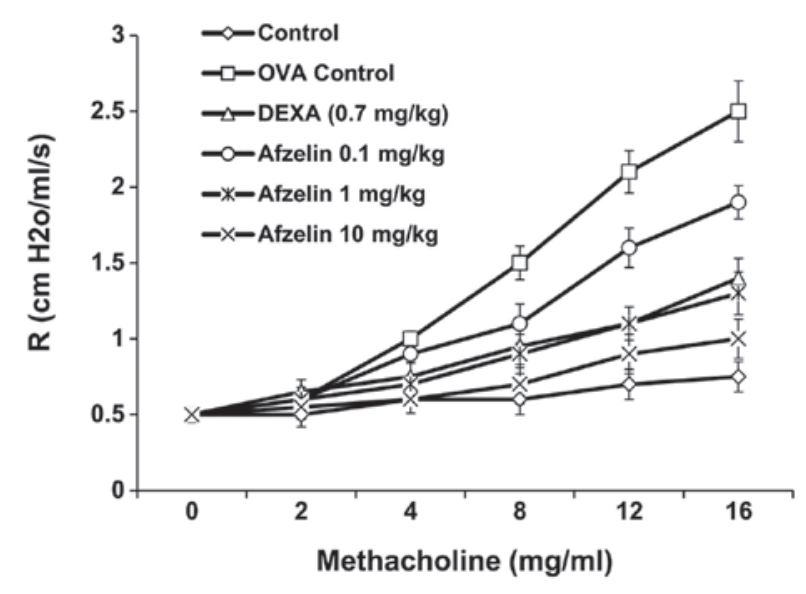

Figure 3. Measurement of airway hyperresponsiveness. Afzelin administration reduces airway hyperresponsiveness in mice as measured by a methacholine dose-responsive cure for airway resistance. Values are expressed as the mean \pm standard error of the mean. ${ }^{*} \mathrm{P}<0.05$, vs. sham and ${ }^{* *} \mathrm{P}<0.01$, vs. sham (Student's t test). Dexa, dexamethasone; OVA, chicken egg albumin.

polyvinylidene difluoride membrane (Bio-Rad Laboratories, Inc.) in transfer buffer using a Mini Transblot electrophoretic transfer cell (Bio-Rad Laboratories, Inc.) for 90-120 min at $150 \mathrm{~V}$. Membranes were blocked in $5 \%$ fat-free dry milk (Abcore) dissolved in Tris-buffered saline and Tween 20. for $2.5 \mathrm{~h}$ at room temperature. Anti-GATA3 and anti-T-bet mouse polyclonal antibodies (1:1,000 dilution; Bio-Rad Laboratories, Inc.) were used to determine expression of their corresponding proteins, and a monoclonal $\beta$-actin antibody was used as the loading control (Sigma-Aldrich) (11). After incubation with the primary antibodies overnight at $4^{\circ} \mathrm{C}$ the membranes were incubated with goat anti-mouse immunoglobulin $\mathrm{G}$ secondary antibody (1:5,000 dilution; Bio-Rad Laboratories, Inc.) for $1 \mathrm{~h}$ at $25^{\circ} \mathrm{C}$. The blots were visualized with a chemiluminescent detection system (ECL; GE Healthcare Australia, Rydalmere, Australia) according to the manufacturer's instructions.

Histological examination. After BALF was obtained, the left lung was removed, fixed in $10 \%$ neutral buffered formalin for $24 \mathrm{~h}$ and then the specimens were dehydrated and embedded in paraffin in a standard manner. In order to perform histological examination, 5- $\mu \mathrm{m}$ sections of fixed embedded
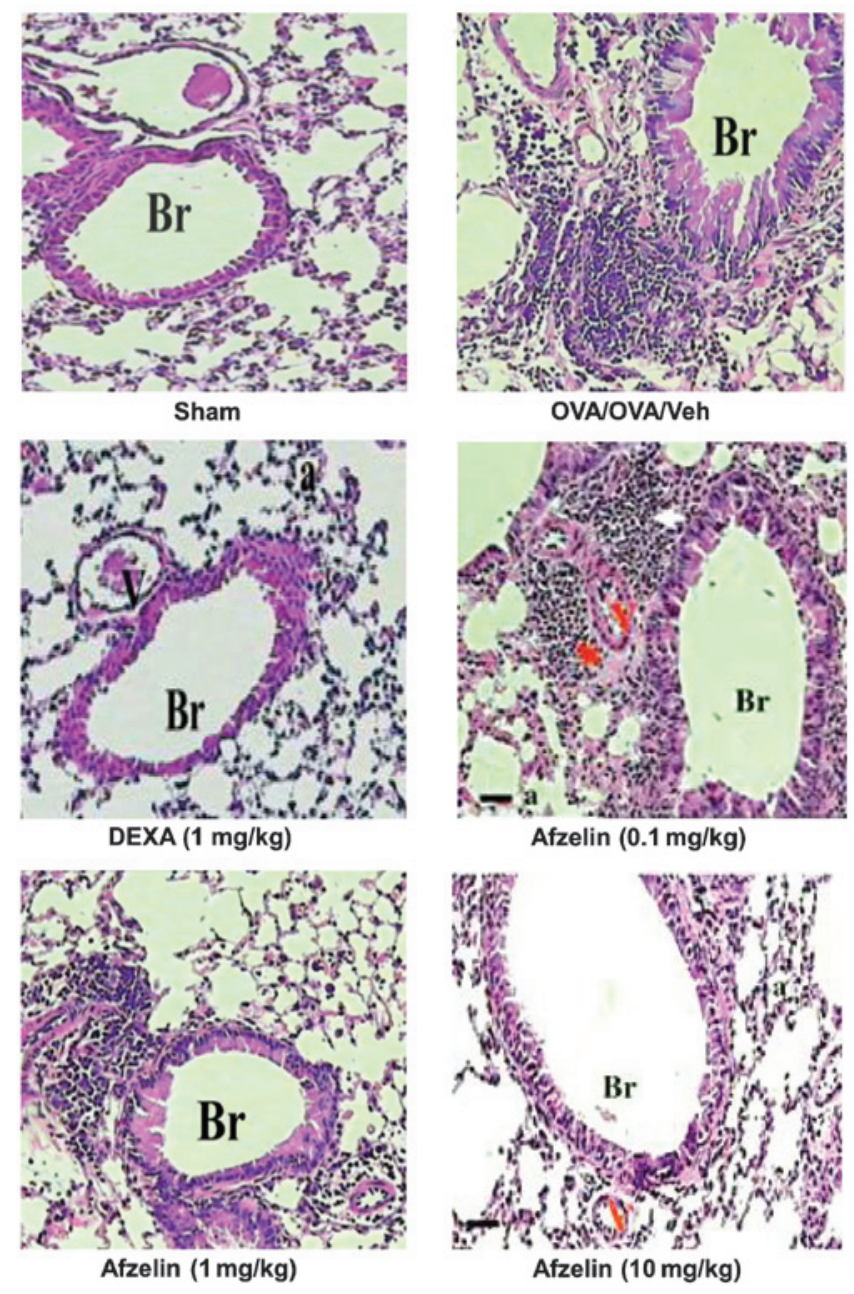

Figure 4. Afzelin treatment significantly reduced airway cellular infiltration as detected by hematoxylin \& eosin staining of lung sections. Dexa, dexamethasone; OVA, chicken egg albumin; Veh, vehicle; Br, bronchiole.

tissues were cut and stained with hematoxylin \& eosin (Abcore) according to routine laboratory procedures (12). Histological analyses were performed by pathologists blinded to the treatment groups. For each mouse, five airway sections, distributed throughout the left lung, were analyzed with the use of a light microscope (MCL-3000) attached to an image-analysis system (Image-Pro Plus 4.0; Media Cybernetics, Minneapolis, MN, USA). The images were 
A

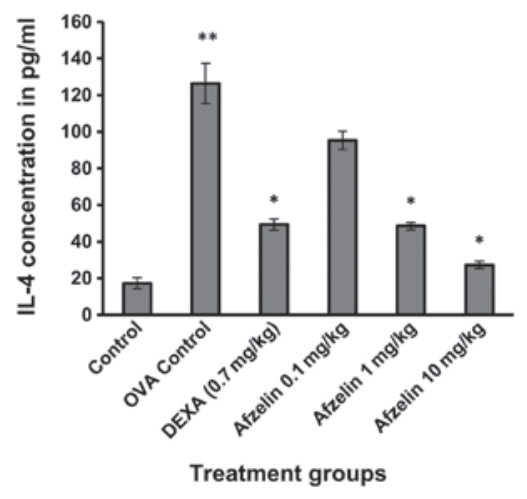

C

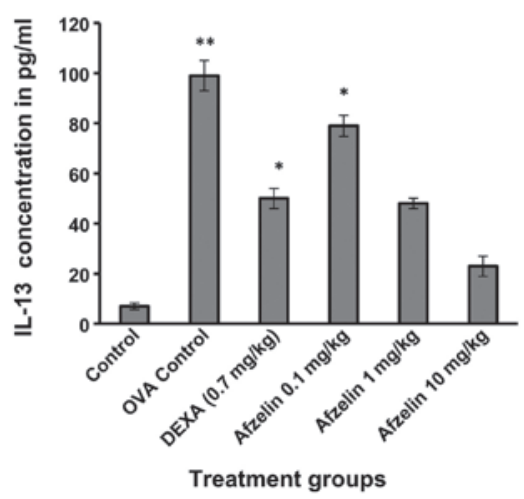

B

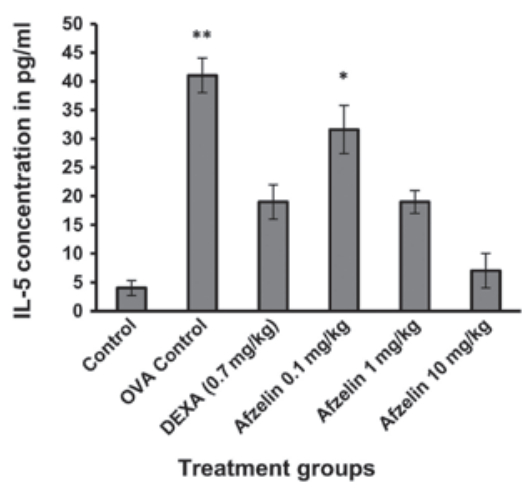

D

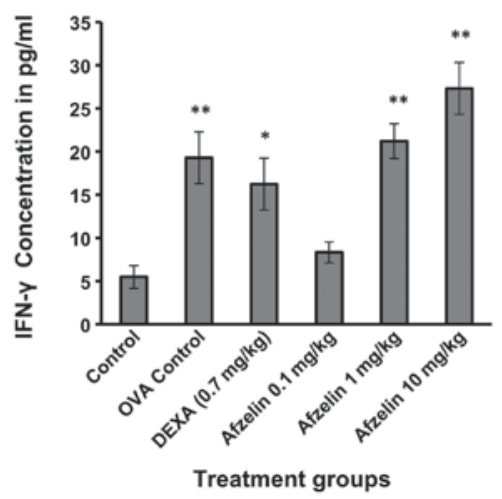

Figure 5. Effect of test compounds on Th2 cytokine release. (A) IL-4; (B) IL-5; (C) IL-13 and (D) IFN- $\gamma$. The levels of IL-4, IL-5 and IL-13 in BAL fluid were quantified by sandwich ELISA and expressed as picogram per milliliter. Values are expressed as the mean \pm standard error of the mean. "P<0.05, vs. sham and ${ }^{* *} \mathrm{P}<0.01$, vs. sham (Student's t test). TH2, T-helper 2; IL, interleukin; IFN, interferon; BAL, bronchoalveolar lavage; Dexa, dexamethasone; OVA, chicken egg albumin.

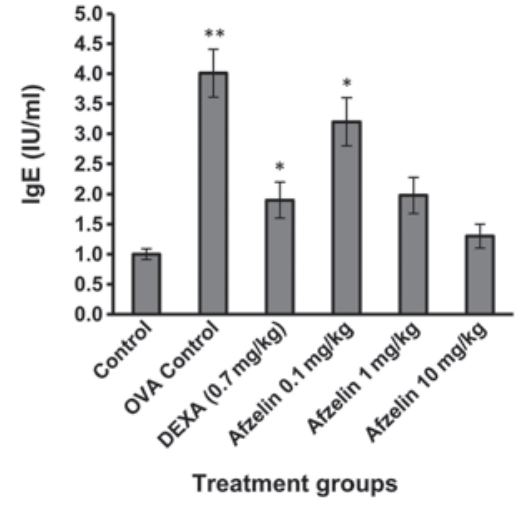

Figure 6. Effect of afzelin on OVA specific IgE release. Values are expressed as the mean \pm standard error of the mean. ${ }^{*} \mathrm{P}<0.05$, vs. sham and ${ }^{* *} \mathrm{P}<0.01$, vs. sham (Student's t test). OVA, chicken egg albumin; IgE, immunoglobulin E.

then cropped and corrected for brightness and contrast, but otherwise were not manipulated (7).

Statistical analysis. Groups were analyzed using a one-way analysis of variance followed by Dunnett's multiple comparison tests to examine differences between OVA-challenged as well as PBS- and afzelin- and Dexa-treated groups. $\mathrm{P}<0.05$ was considered to indicate a statistically significant difference. Values are presented as the mean \pm standard error of the mean for each group.

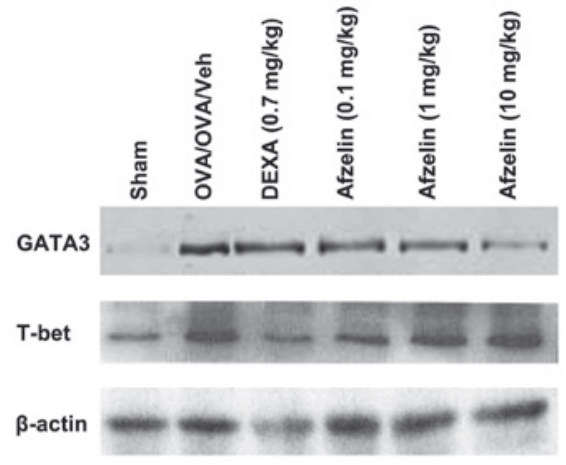

Figure 7. Effect of the test compounds on GATA3 and T-bet expression using western blot analysis. Dexa, dexamethasone; OVA, chicken egg albumin; GATA3, GATA-binding protein 3.

\section{Results}

Afzelin decreases AHR in experimental asthma. To examine the effect of afzelin on AHR, airway resistance was measured in anaesthetized mice by invasive whole-body plethysmography. No significant difference was found in baseline airway resistance among the six groups. The airway resistance generated by administration of Mch at doses of $0-16 \mathrm{mg} / \mathrm{ml}$ significantly increased in the OVA and afzelin $(0.1 \mathrm{mg} / \mathrm{kg})$-treated groups. However, the control, Dexa- and afzelin (1 and $10 \mathrm{mg} / \mathrm{kg}$ )-treated groups exhibited a sharp decrease in airway resistance (Fig. 3). 
Afzelin attenuates airway inflammation. Apart from macrophages, only few inflammatory cells were detected in the control group. However, a significant increase in total cell number was observed in OVA-sensitized and challenged animals, when compared with those in the control mice. The effect of afzelin on allergen-induced inflammatory cell infiltration was assessed in animals treated with three different doses of afzelin. As shown in Table I, afzelin at 1 and $10 \mathrm{mg} / \mathrm{kg}$ suppressed allergen-induced inflammatory cell infiltration. However, in the case of the $0.1 \mathrm{mg} / \mathrm{kg}$-treated group, infiltration of inflammatory cells was not reduced. The anti-inflammatory effect of afzelin was further demonstrated by histological examination of hematoxylin \& eosin-stained sections (Fig. 4). A marked affluence of inflammatory cells into the airway was observed in OVA-sensitized/challenged mice, but not in the PBS-treated control mice. Mice treated with afzelin exhibited a marked diminution in inflammatory cell infiltration into the airways.

Afzelin affects Th1 and Th2 cytokine release. Measurement of the Th2 cytokines IL-4, -5 and -13 was performed in the serum collected from the mice. Mice treated with the test compound afzelin demonstrated no significant change in cytokine release when compared with those in the control at doses of 1 and $10 \mathrm{mg} / \mathrm{kg}$ (Fig. 5). However, cytokine levels measured in the $0.1 \mathrm{mg} / \mathrm{kg}$-treated group of animals exhibited a significant variation from the control group. Afzelin increased the release of IFN- $\gamma$, a Th1 cytokine, indicating that it affects T-cell differentiation, which was further supported by its effect on GATA3 and T-bet.

Afzelin reduces OVA-specific IgE levels. OVA-specific IgE levels were elevated in the OVA group when compared with those in the control group (Fig. 6). Treatment with afzelin (1 and $10 \mathrm{mg} / \mathrm{kg}$ ) demonstrated no significant change in OVA-specific IgE levels as compared with those in the control group.

Afzelin alters the expression of T-bet and GATA-3 in the lungs. Expression levels of T-bet and GATA-3 in the lungs were altered in OVA control mice, Dexa-treated mice and afzelin-treated mice. However, no change was observed in the expression levels of any of these proteins in control animals. Treatment with afzelin increased expression of T-bet, while at the same time decreasing GATA-3 expression in a dose-dependent manner (Fig. 7).

\section{Discussion}

In the present study, the effects of afzelin on allergen-induced airway inflammation and immune response in acute experimental asthma were assessed. It was found that administration of afzelin markedly reduced Th2 cytokine levels and OVA-specific IgE, and suppressed airway inflammatory cell infiltration induced by allergens, resulting in a decreased number of eosinophils and total inflammatory cells in BALF. Lung histology validated the effect of afzelin on airway inflammation. These findings suggested that afzelin is an anti-asthmatic agent and may be beneficial for the treatment of allergic asthma.
It is widely accepted that $\mathrm{T}$ cells have an important role in the injurious lung immune responses associated with asthma $(13,14)$. CD4 ${ }^{+}$Th cells can be divided into Th1 and Th2 groups, functionally based on the various types of cytokine they produce. The different patterns of T-cell differentiation generate the different inflammatory effectors and those inflammatory effectors are correlated with the extent and type of damage observed in the airways (15-17). Under normal physiological conditions, the ratio of Th1 to Th2 cells is maintained at an appropriate level. Once the balance between Th1 and Th2 is disrupted, disease may occur (18). The two major Th-specific transcription factors T-bet and GATA-3, which regulate the expression of the cytokine genes, are characteristics of Th1 or Th2 and have crucial roles in Th-cell differentiation. It has been reported that a change in the T-bet/GATA-3 ratio reflects a change in the Th1/Th2 balance (18-20). Therefore, the T-bet/GATA-3 ratio may be used to evaluate the immune balance of Th1/Th2 responses in asthma (4). In addition, increased IL-4 production is correlated with excessive Th2-cell responses and increased IFN- $\gamma$ levels are associated with excessive Th1 cell responses (21). In the present study, the ratio of T-bet to GATA-3 decreased in the asthma group compared with that in the control group and was partly reverted in the afzelin treatment groups. At an equal pace, IL-4 production was depressed and IFN- $\gamma$ levels increased in the treatment groups. This change was more prominent in the 1 and $10 \mathrm{mg} / \mathrm{kg}$ afzelin-treated groups. Treatment with Dexa had similar effects to those of $1 \mathrm{mg} / \mathrm{kg}$ afzelin. The pathophysiology of AHR is complex as numerous factors contribute to its development. Allergen-induced airway inflammation is important among these factors, and Th2 cytokines, particularly IL-4, are critical in allergic inflammation and development of AHR (22).

In the present study, no significant difference was found in baseline airway resistance among the six groups. The airway resistance generated by administration of $\mathrm{Mch}$ at $30-270 \mu \mathrm{g} / \mathrm{kg}$ was significantly increased in the OVA group and the afzelin $(0.1 \mathrm{mg} / \mathrm{kg})$-treated group. However, the control group, Dexa- and afzelin (1 and $10 \mathrm{mg} / \mathrm{kg}$ )-treated groups revealed a sharp decrease in airway resistance. In conclusion, the present study indicated that afzelin is promising as a beneficial medication for the treatment of asthma through ameliorating allergic responses.

\section{References}

1. Global Initiative for Asthma (GINA), Global Strategy for Asthma Management and Prevention, GINA, 2014. http://www. ginasthma.org. Accessed December 15, 2014.

2. Masoli M, Fabian D, Holt S and Beasley R: The global burden of asthma: executive summary of the GINA Dissemination Committee report. Allergy 59: 469-478, 2004.

3. Zhu J, Yamane H, Cote-Sierra J, Guo L and Paul WE: GATA-3 promotes Th2 responses through three different mechanisms: induction of Th2 cytokine production, selective growth of Th 2 cells and inhibition of Th1 cell-specific factors. Cell Res 16: 3-10, 2006.

4. Nath P, Leung SY, Williams AS, et al: Complete inhibition of allergic airway inflammation and remodelling in quadruple IL-4/5/9/13-/-mice. Clin Exp Allergy 37: 1427-1435, 2007.

5. Gong JH, Shin D, Han SY, Kim JL and Kang YH: Kaempferol suppresses eosionphil infiltration and airway inflammation in airway epithelial cells and in mice with allergic asthma. J Nutr 142: 47-56, 2012 
6. DiantiniA,SubarnasA,LestariK,etal:Kaempferol-3-O-rhamnoside isolated from the leaves of Schima wallichii Korth. inhibits MCF-7 breast cancer cell proliferation through activation of the caspase cascade pathway. Oncol Lett 3: 1069-1072, 2012.

7. Mabalirajan U, Dinda AK, Kumar S, et al: Mitochondrial structural changes and dysfunction are associated with experimental allergic asthma. J Immunol 181: 3540-3548, 2008.

8. Philippe P, Pierre C, Danuta R, Bart NL and Martin O: Protein tyrosine phosphatases regulate asthma development in a murine asthma model. J Immunol 182: 1334-1340, 2009.

9. Du Q, Zhou LF, Chen Z, Gu XY, Huang M and Yin KS Imiquimod, a toll-like receptor 7 ligand, inhibits airway remodelling in a murine model of chronic asthma. Clin Exp Pharmacol Physiol 36: 43-48, 2009.

10. Jones CG, Daniel Hare J and Compton SJ: Measuring plant protein with the Bradford assay: 1. Evaluation and standard method. J Chem Ecol 15: 979-992, 1989.

11. Seung-Hyung K, Bok-Kyu K and Young-Cheol L: Antiasthmatic effects of Hesperidin, a potential Th2 cytokine antagonist, in a mouse model of allergic asthma. Mediators Inflamm 2011: 485402, 2011.

12. Borges O, Borchard G, Sousa AD, Junginger HE and Cordeiro-da-Silva A: Induction of lymphocytes activated marker CD69 following exposure to chitosan and alginate biopolymers. Int J Pharm 337: 254-264, 2007.

13. Anderson GP and Coyle AJ: TH2 and 'TH2-like' cells in allergy and asthma: pharmacological perspectives. Trends Pharmaco Sci 15: 324-332, 1994

14. Coyle AJ, Le Gros G, Bertrand C, et al: Interleukin-4 is required for the induction of lung Th2 mucosal immunity. Am J Respir Cell Mol Biol 13: 54-59, 1995.
15. Morrisey EE, Ip HS, Lu MM and Parmacek MS: GATA-6: a zinc finger transcription factor that is expressed in multiple cell lineages derived from lateral mesoderm. Dev Biol 177: 309-322, 1996.

16. Arceci RJ, King AA, Simon MC, Orkin SH and Wilson DB: Mouse GATA-4: a retinoic acid-inducible GATA-binding transcription factor expressed in endodermally derived tissues and heart. Mol Cell Biol 13: 2235-2246, 1993

17. Cho SH, Stanciu LA, Holgate ST and Johnston SL: Increased interleukin-4, interleukin-5, and interferon-gamma in airway CD4+ and CD8+ T cells in atopic asthma. Am J Respir Crit Care Med 171: 224-230, 2005.

18. Szabo SJ, Sullivan BM, Peng SL and Glimcher LH: Molecular mechanisms regulating Th1 immune responses. Ann Rev Immunol 21: 713-758, 2003.

19. Szabo SJ, Sullivan BM, Stemmann C, Satoskar AR, Sleckman BP and Glimcher LH: Distinct effects of T-bet in Th1 lineage commitment and IFN-gamma production in CD4 and CD8 T cells. Science 295: 338-342, 2002.

20. Harrington LE, Hatton RD, Mangan PR, et al: Interleukin 17-producing CD4+ effector T cells develop via a lineage distinct from the T helper type 1 and 2 lineages. Nat Immunol 6: 1123-1132, 2005.

21. Zhang DH, Cohn L, Ray P, Bottomly K and Ray A: Transcription factor GATA-3 is differentially expressed in murine Th1 and Th2 cells and controls Th2-specific expression of the intrleukin-5 gene. J Biol Chem 272: 21597-21603, 1997.

22. Cohn L, Tepper JS and Bottomly K: IL-4-independent induction of airway hyperresponsiveness by Th2, but not Th1, cells. J Immunol 161: 3813-3816, 1998 